\section{COVID vaccine results pour in}

On 20 July, results from clinical trials of two early-stage COVID-19 vaccines, one developed by University of Oxford and AstraZeneca and another by the Chinese company CanSino Biologics, were reported in the Lancet. Oxford's simian adenovirus-vectored vaccine (ChAdOx1 nCoV-19) and CanSino's adenovirus-5-vectored vaccine both elicited neutralizing antibodies and T-cell immune responses to the SARS-CoV-2 virus. (CanSino, based in Tianjin, was the first vaccine developer to publish data from a smaller phase 1 trial, in May.) These publications join Moderna's mRNA vaccine report from mid-July in the New England Journal of Medicine. Because of different assays used, comparisons among the vaccines are difficult. In broad terms, the two adenovirus-vectored programs gave similar results - upwards of 80-90\% of participants marshalled neutralizing antibody and spike-protein-specific T cell responses within 28 days - whereas the Moderna vaccine study reported only neutralizing antibody responses after vaccination. In the single-blind, randomized, placebo-controlled Oxford trial, immune responses lasted the full 56 days of the trial. The researchers inoculated a small cohort of ten people with a second dose of the COVID-19 vaccine after 28 days, which upped the antibody response but not the T-cell one, in keeping with previous observations. Participants in both trials had only mild flu-like symptoms, typically seen with viral-vector-mediated vaccines.

Although the results are promising, both trials involved relative young and ethnically homogenous people; neither of these represent demographics most affected by COVID-19. More diverse populations are included in Cambridge-based AstraZeneca's phase 3 trials of ChAdOx1 nCoV-19 taking place in South Africa and Brazil. Also on 20 July, Pfizer and German company BioNtech of Mainz published early results in an online preprint server of their mRNA vaccine candidate BNT162b1, showing dose-dependent neutralizing antibody responses and $\mathrm{CD} 4^{+}$and $\mathrm{CD} 8^{+} \mathrm{T}$ cell responses against the receptor-binding domain of SARS-CoV-2. Ultimately, though, although all of these potential vaccines have shown to engage the immune system, no vaccine developer has yet provided definitive data demonstrating protection from SARS-CoV-2 infection.

Published online: 5 August 2020

https://doi.org/10.1038/s41587-020-0647-4 the drug as a frontrunner among the anti-GM-CSF mAbs under development. Mavrilimumab eliminated deaths in the treated group, with $100 \%$ attaining clinical improvement by day 28 , as compared with only $65 \%$ in the comparison group. According to a presentation planned for the European League Against Rheumatism (EULAR) 2020 Digital Congress in August, Kiniksa is moving forward on the basis of this data with a multicenter, double-blind, randomized, placebo-controlled study.

Although GM-CSF blockade is an area of high trial activity, few results have as yet been published in the peer-reviewed literature. Other anti-GM-CSF antibodies in clinical trials for COVID-19 include Humanigen's lenzilumab (an engineered, non-fucosylated recombinant human IgG1 $\mathrm{mAb}$ ), which is in phase 3 trials, and Shanghai-based I-Mab's TJ003234 (a humanized IgG1 mAb), which is in phase 2 testing. Three other fully human IgG1 mAbs against GM-CSF are undergoing efficacy testing for COVID-19: Basel, Switzerland-based Roivant's gimsilumab; otilimab, from Planegg, Germany-based MorphoSys and Brentford, UK-based GlaxoSmithKline; and Oxford-based Izana's namilumab.

All things considered, clinical management of COVID-19 will likely require several different therapeutic strategies. "The idea that one biological therapy may turn out to be superior to others is probably not accurate" says Simon Jones of the Systems Immunity Research Institute, School of Medicine at Cardiff University in the UK. Different patients with the same underlying condition, such as rheumatoid arthritis, often show differing responses to specific biological therapies. "This is also probably reflected in patients with COVID-19," he says.

As an adjunct to these biologic approaches, small molecules have the advantage of being able to inhibit several cytokines at once. Cambridge, UK-based AstraZeneca's Calquence (acalabrutinib), approved for treating chronic lymphocytic leukemia, targets Bruton's tyrosine kinase (BTK), which is upstream of several inflammatory cytokines, such as IL-1, IL- 6 , and TNF- $\alpha$. An off-label trial published in Science Immunology showed that Calquence reduced patients' requirement for oxygen and normalized markers of inflammation. Levels of BTK were elevated in peripheral blood monocytes isolated from patients with COVID-19 compared with cells from healthy volunteers. A possible advantage to targeting BTK is that it is "specific for the type of cell - the macrophage - that is a leading cause of the cytokine surge," says Louis Staudt at the Center for Cancer Research at the US National Cancer Institute, an author on the publication. As such,
Calquence might have fewer side effects than other multi-cytokine blockers, such as JAK-STAT inhibitors, currently in clinical trials that modulate cytokine activity downstream of their receptors. On the basis of this result, AstraZeneca plans to test Calquence in larger trials.

If the preferred strategy is to tackle many cytokines at once, why not use steroids? Indeed, the steroid dexamethasone reduced deaths by one-third in patients on a ventilator and by one-fifth in patients receiving oxygen, as reported in a preprint in June from the large RECOVERY trial. However, dexamethasone only made a difference to patients seriously ill with COVID-19; in individuals not sick enough to be on respiratory support, dexamethasone did not improve survival. The aim of a therapy should be to "prevent people getting to ventilator stage, before damage such as organ disfunction, clotting disorders and secondary infections manifest," says Wyndham Wilson, senior investigator at the US National Cancer Institute. He adds that dexamethasone might be affecting inflammation within the lung itself and still do little to dampen the cytokine storm. When to intervene with a sledgehammer approach, such as dexamethasone, is just as critical. Starting a treatment that stifles the immune system too early in the disease course runs the risk of promoting viral replication because the very cytokines that are part of the surge also are vital in the host's defense against the virus.

To track when a patient's inflammatory response becomes life-threatening can be done with routine clinical tests, such as measurements of ferritin or C-reactive protein. And measuring IL-6 is relatively straightforward. But these types of analyses give only a blurry picture. "They are an index of inflammation that don't really tell us much about the direction of the pathology", says Jones. Markers, of say, endothelial damage, abnormal coagulation or lymphopenia may be better indicators of severe disease, he adds. In this respect, blood profiling and immune cell and biomarker studies may provide a clearer picture (Box 1).

Overall, the path to therapeutic success in COVID-19 will lie somewhere between stopping the hyperinflammation before the patient becomes very sick and allowing the body to eliminate persisting virus. "We're trying to do two things at the same time" says Merad. "This is a conundrum we are all facing."

\section{Charlotte Harrison \\ Canterbury, UK}

Published online: 5 August 2020

https://doi.org/10.1038/s41587-020-0634-9 